\title{
Lack of Treatment Persistence and Treatment Nonadherence as Barriers to Glycaemic Control in Patients with Type 2 Diabetes
}

\author{
Bruno Guerci (D) - Neha Chanan · Simarjeet Kaur · Juan Guillermo Jasso-Mosqueda • \\ Elisheva Lew
}

Received: December 20, 2018 / Published online: March 8, 2019

(C) The Author(s) 2019

\begin{abstract}
Treatment persistence (continuing to take medication for the prescribed period) and treatment adherence (complying with the prescription in terms of drug schedules and dosage) are both important when treating chronic diseases such as type 2 diabetes (T2D). They can be indicators of patient satisfaction with treatment. In $\mathrm{T} 2 \mathrm{D}$, the achievement of optimal outcomes requires both persistence with and adherence to prescribed therapy. Poor persistence with and adherence to T2D medication can have profound consequences for the patient, including non-achievement of glycaemic goals and an increased risk of long-term complications and mortality. Therefore, poor treatment persistence and adherence may also have economic consequences, including increased healthcare resource utilization and healthcare costs. Treatment persistence and
\end{abstract}

Enhanced Digital Features To view enhanced digital features for this article go to https://doi.org/10.6084/ m9.figshare.7752116.

B. Guerci $(\bowtie)$

Brabois Hospital and CIC INSERM ILCV, University

Hospital of Nancy, Vandoeuvre Lès Nancy, France

e-mail: b.guerci@chru-nancy.fr

N. Chanan $\cdot$ S. Kaur

Parexel Access Consulting, Mohali, India

J. G. Jasso-Mosqueda · E. Lew

Sanofi Aventis, Chilly-Mazarin, France adherence are affected by several factors, including the mode of administration, administration frequency/regimen complexity, and patient expectations. The aims of this review are as follows: to provide an overview of persistence with and adherence to different antidiabetes therapies for patients with T2D in the real-world setting; examine factors contributing to poor treatment persistence and adherence; and assess available data on the impact of poor treatment persistence and/or adherence on clinical and economic outcomes. Numerous potential targets for improving treatment persistence and/or adherence are identified, including developing less complex treatment regimens with lower pill burdens or less frequent injections, improving the convenience of drug-delivery systems, such as the use of insulin pen devices rather than the conventional vial and syringe, and developing therapies with an improved safety profile to alleviate patient fears of adverse effects, such as weight gain and risk of hypoglycaemia.

Funding: Sanofi.

Keywords: Adherence; Diabetes; Persistence; Type 2 diabetes

\section{INTRODUCTION}

When treating type 2 diabetes (T2D), as with any chronic disease, the achievement of 
optimal therapeutic outcomes relies on both treatment persistence and treatment adherence. In real-world studies of patients with T2D, persistence with and adherence to antidiabetes therapies are defined and evaluated in many different ways. Treatment persistence is the continuation of therapy over time. It is often measured by the availability of the drug, expressed as the continuous filling of prescriptions (e.g. as the percentage of patients still obtaining the drug at 12 months) or alternatively as "the duration of time from initiation to discontinuation of therapy" [1]. Treatment adherence is defined as "the extent to which a patient acts in accordance with the prescribed interval and dose of a dosing regimen" [1]. This is based on the principle of conformity to a rule enacted by others. Understanding the rule is not necessary. In medical practice, this is understood as strict compliance with prescriptions in terms of schedules and dosages. Direct measurement of treatment adherence is difficult and is often expressed indirectly using refill records as either the medication possession ratio (MPR) or the proportion of days covered (PDC). Although the exact formula used to calculate MPR varies according to source, it may be defined as the sum of the days' supply for all fills of a given drug in a particular time period divided by the number of days in the time period. Patients who routinely refill their medications early will have an inflated MPR, whereas population estimates of MPR may also be falsely elevated unless individual adherence is capped at $100 \%$. PDC, in contrast, is a more conservative measure of refill record-based adherence [2]. Instead of adding the days' supplied in a given period, PDC considers only the days that are "covered". The use of treatment adherence complements that of treatment persistence by including the notion of a drug amount necessary for the functioning of the treatment. Measuring treatment adherence from databases is complex, as compliance with a dosing schedule cannot be assessed; compliance with dosing is generally replaced with an assessment of the availability of a sufficient quantity of the drug to be able to comply with dosing. Adherence is a qualitative tool in its own right.
Pharmacological approaches to achieving glycaemic control in patients with T2D usually involve a sequential programme of treatment intensification from monotherapy with oral medication, dual and triple therapy with a combination of oral therapies or oral plus injectable agents, and combination injectable therapy $[3,4]$. Although persistence with and adherence to T2D medications vary according to the treatment modality, lack of persistence and adherence in general act as a substantial barrier to the achievement of glycaemic control. Persistence and adherence are affected by several factors, including the mode of administration, administration frequency/ regimen complexity, and patient expectations. Regarding the mode of administration, persistence and adherence are generally better for oral medications compared with injected medications. However, persistence and adherence may vary even among different oral antidiabetes drugs (OADs) classes and for different insulin regimens, indicating that factors other than administration route are also important [5-8]. In the case of insulin regimen, for example, a higher daily insulin dose and greater regimen complexity may contribute to poorer treatment adherence $[7,8]$. Persistence and adherence are also influenced by the frequency of administration, with fewer daily insulin injections and fixed-ratio combinations that reduce pill counts having beneficial effects on adherence $[5,9]$. Mashitani et al. [10] showed that patients with T2D who take more injections are more likely to omit insulin injections. Mashitani et al. [11] also reported that the risk of lower adherence compared with higher adherence increased as the number of injections increased (relative risk [RR] 0.98; 95\% confidence interval [CI] 0.65-1.46). Fear of injections and adverse effects such as hypoglycaemia and weight gain have all been reported as reasons for nonadherence to antihyperglycaemic medications [12-14]. They also vary according to patient expectations, such as the effects of medication on weight [15]. Although the simplification of regimens associated with the use of premixed insulins can have a positive effect on adherence, other factors concerning patient satisfaction may come into play. Additionally, studies comparing 
premixed and basal insulin regimens have produced mixed results concerning the relative reduction of glycated haemoglobin (HbA1c), but most studies have shown an increased risk of hypoglycaemic events and weight gain with premixed insulin [16, 17], which may have a negative impact on adherence and persistence.

The aims of this review article are to provide an overview of persistence with and adherence to different antidiabetes therapies in patients with T2D in the real-world setting, to examine factors contributing to poor treatment persistence and adherence, and to assess available data on the impact of poor treatment persistence and/or adherence on clinical and healthcare utilization. This review is based on previously conducted studies and does not contain any studies with human participants or animals performed by any of the authors.

\section{LITERATURE SEARCH}

A literature search was conducted in October 2015 using EMBASE and PubMed to identify sources to inform this review article. The literature search was limited to English language articles within the timeframe of November 2013 to October 2015. Although this timeframe excluded older articles such as those relating to adherence to sulfonylureas and, conversely, selected for modern treatment options such as glucagon-like peptide-1 receptor agonists (GLP1 RA), the intention was to review topical literature relating to persistence and adherence in patients with T2D. However, when data were limited, the publication timeframe criterion was expanded. A reviewer screened the abstracts for inclusion in a full text review and then screened full text references to identify any additional publications with relevant information for inclusion in the targeted literature review, and hence in this review article. The literature search was complemented in February 2018 by internet searches performed by hand, which included literature published between November 2015 and December 2017.

\section{PERSISTENCE WITH AND ADHERENCE TO ANTIDIABETES THERAPIES}

\section{Oral Antidiabetes Drugs}

Patients with T2D are generally more persistent with their treatment if they are treated with OADs compared with injectable agents. A retrospective study in the US followed a large cohort $(N=51,771)$ of patients with T2D who were uncontrolled on 2 OADs and who added either a third OAD or an injectable agent (a GLP-1 RA or insulin) [18]. During the 2-year follow-up period, treatment persistence (defined as the percentage of patients remaining on the index drug treatment until the end of year 1 and year 2 of follow-up) was higher for patients with T2D who initiated a third OAD (72\%) than for those who initiated either a GLP-1 RA (36\%) or insulin (57\%). However, it was noted that higher treatment persistence among patients adding a third OAD did not translate into better glycaemic control in this patient group. Additionally, annual all-cause healthcare costs declined in the group who added insulin but increased in the other groups.

Compared with insulin and OADs that have an insulin-dependent mechanism of action such as sulfonylureas and meglitinides, the risk of hypoglycaemia is relatively small with metformin, thiazolidinediones, and dipeptidyl peptidase-4 (DPP-4) inhibitors [19]. Together with weight gain and gastrointestinal side effects, hypoglycaemia is an important attribute for patients in their OAD preferences [20]. Unsurprisingly, therefore, hypoglycaemia is associated with a higher risk of treatment discontinuation in patients with T2D [19]. Studies have also shown that patients with recent hypoglycaemia, or those who experience moderate or worse symptoms of hypoglycaemia, are more likely to have poor treatment adherence compared to patients with less recent or no prior hypoglycaemia, or those with mild hypoglycaemia symptoms [21, 22].

Among patients with T2D who initiate a fixed-dose combination of DPP-4 inhibitor plus metformin following switch from dual therapy, 
the resulting improvements in glycaemic control and/or reductions in gastrointestinal side effects are speculated as being related to improved adherence post-switch [23, 24]. Other factors associated with adherence to oral medications include family support among children and adolescents with T2D [25], and self-efficacy and perceptions of illness among adults with T2D [26].

\section{Basal Insulin}

Treatment nonadherence and poor treatment persistence are important barriers to overcome for the achievement and maintenance of glycaemic control in patients with diabetes once insulin treatment is initiated. Reported rates of adherence to insulin vary from $30 \%$ to $86 \%$ depending on the patient population and the measure of treatment adherence used $[12,14,27]$. Determining treatment persistence and adherence is particularly difficult when evaluating insulin. Insulin is prescribed in fixed amounts, rather than in 1- or 3-month supplies, as is common with oral therapies. A box of injection pens contains 1500 units, and the starting dose is often 10 units. Therefore, although an initial prescription might last 150 days, patients often change their dose while on treatment, making this unlikely. Without information regarding a patient's dose, treatment persistence cannot be evaluated from claims data. Treatment adherence suffers from the same challenges because an evaluation of adherence also requires information on the intended dose.

Persistence with and adherence to initial basal insulin have been shown to be relatively high in insulin-naive patients with diabetes. A recent French study evaluated treatment persistence among 1969 insulin-naive patients with T2D who initiated basal insulin (61.8\%), basal and fast-acting insulin (15.0\%), or other insulin regimens $(23.2 \%)$ [28]. During the first 12 months after treatment initiation, $24.9 \%$ of patients discontinued insulin therapy; however, discontinuation was lower for basal insulin (18.4\%) and basal and fast-acting insulin $(17.7 \%)$ compared with other insulin regimens
(39.6\%) [28]. Elderly (aged $\geq 65$ years) insulinnaive patients with T2D initiating basal insulin therapy in the US were also shown to have relatively high rates of persistence with therapy at 12 months (>50\%) in the real-world setting [29] and tended to be adherent to therapy $(>60 \%)$, although treatment persistence and treatment adherence are greatly influenced by the type of insulin and delivery device used [30].

Insulin pen devices have been shown to offer improved treatment persistence and adherence compared with traditional insulin vial-and-syringe delivery $[14,29,30]$. In a study of the effects of insulin delivery systems in elderly patients with T2D, it was found that patients initiating insulin glargine using a pen device were significantly more persistent $(58.5 \%$ vs $50.8 \%$ at 12 months, respectively; $P<0.0001$ ) and adherent (adjusted MPR $\geq 0.08 ; 71.5 \%$ vs $63.1 \%$, respectively; $P<0.0001$ ) than patients who initiated treatment using vial-and-syringe delivery [29].

Another study followed three propensitymatched cohorts of elderly patients with T2D who were either insulin naive, initiating insulin glargine with a pen device or with vial and syringe, or were already administering insulin glargine by vial and syringe [30]. At the 1-year follow-up, pen initiators were significantly more persistent than vial initiators $(58.2 \%$ vs $50.8 \%$, respectively; $P<0.0001)$ and were significantly more adherent (adjusted MPR 0.69 vs 0.64 , respectively; $P<0.0001)$. Pen initiators were also significantly more persistent and adherent than patients who continued to use the vial and syringe.

A systematic review of factors affecting realworld treatment adherence in patients with type 1 diabetes (T1D) or T2D also identified several studies showing significantly better treatment adherence among pen users compared with vial-and-syringe users, both for patients initiating insulin therapy and for patients switching from vial-and-syringe delivery to a pen device [14].

In a US propensity-matched cohort analysis of data from a managed-care claims database, significantly more patients with T2D initiating insulin therapy with insulin glargine compared 
with insulin detemir (both administered via disposable pen) persisted with therapy during the 1 -year follow-up period $(64.8 \%$ vs $50.9 \%$, respectively; $P<0.0001)$ [31]. Treatment adherence was also significantly higher during follow-up in the insulin glargine cohort than in the insulin detemir cohort (MPR 0.48 vs 0.44 , respectively, $P<0.0049$; adjusted MPR 0.70 vs 0.63 , respectively; $P<0.0001$ ).

A US analysis of two large administrative claims databases investigated the effect of switching between insulin glargine and insulin detemir on clinical and economic outcomes in patients with T2D [32]. During the 1-year follow-up period, patients who were previously treated with insulin glargine and who switched to insulin detemir had significantly lower treatment persistence and adherence than patients who remained on insulin glargine. Furthermore, $33-40 \%$ of patients in the insulin glargine group restarted insulin glargine. Conversely, patients who were previously treated with insulin detemir and who switched to insulin glargine had numerically (but not significantly) higher treatment persistence and adherence than patients who remained on insulin detemir. Although this study did not evaluate dose frequency, insulin detemir is more likely to be administered twice daily compared to insulin glargine, and the authors also noted a higher rate of use of rapid-acting insulins among patients who switched to insulin detemir [32]. Both of these factors might potentially explain the lower persistence and adherence rates associated with switching to insulin detemir.

In two German studies, insulin glargine was associated with significantly higher treatment persistence than insulin detemir and neutral protamine Hagedorn $(\mathrm{NPH})$ insulin in patients with T2D [33, 34]. In patients treated with basal insulin-supported oral therapy, 2-year treatment persistence was 65,53 , and $59 \%$ with insulin glargine, insulin detemir, and NPH insulin, respectively $(P<0.001)$ [33]. In the second study, very similar results were obtained for patients treated with a combination of basal insulin and OADs; 65,53 , and $59 \%$ of patients treated with insulin glargine 100 units $/ \mathrm{mL}$, insulin detemir, and NPH insulin, respectively, persisted with the initially prescribed therapy for over 2 years $(P<0.001)$ [34].

\section{Combination/Multi-injection Therapy}

T2D is a progressive disease for which treatment aims to not only achieve and maintain acceptable glycaemic control but also prevent complications. Therapy therefore frequently includes medications for comorbid disorders such as hypertension and dyslipidaemia, whereas the progression of T2D itself is associated with the use of combination OADs and injectable treatments administered as monotherapy or as part of increasingly complex multi-injection regimens $[35,36]$. As the complexity of the medication regimen increases over time, so too does the burden to the patient, and with it an increased risk of poor treatment persistence and adherence. The nature of this burden is multifactorial and differs for the individual patient, but could relate for example to the number of prescribed agents and dosing frequency, side effects, medication costs, and injection concerns $[14,37]$.

A prospective survey study in Germany showed that a high pill count in antidiabetes therapy constitutes a large burden for patients with T2D, and indicated that physicians prefer to prescribe fixed-dose combinations to reduce their patients' pill burden [38]. Furthermore, a US claims database study found that patients with T2D using a fixed-dose combination of OADs had $21 \%$ lower odds of nonpersistence and $28 \%$ higher odds of adherence compared with those using non-fixed-dose combination therapy [23]. A US database analysis by Sussman et al. [39] suggested that treatment persistence may be lower in patients with T2D who initiate the components of non-fixed-dose combination therapy sequentially compared with those who initiate the components concurrently.

Fear and anxiety related to injections-and more specifically, fear of insulin therapy-have also been reported as a barrier to treatment persistence and adherence $[14,40]$. The questionnaire-based DAWN2 ${ }^{\mathrm{TM}}$ study of patients with T1D or T2D revealed that fear of hypoglycaemia is a major concern for patients, with 
$55.5 \%$ of patients overall reporting that they are "very worried about the risk of hypoglycaemia" [40] and $42 \%$ of insulin-treated T2D patients reporting experiencing hypoglycaemia several times a month [41]. Although a Spanish study suggests that dosing irregularities are less prevalent overall in Spain than in other European countries, fear of nocturnal hypoglycaemia was reported by $24 \%$ of patients, and a reduction in the risk of hypoglycaemia was reported as the main reason for the last time that patients intentionally missed, mistimed, or reduced a basal insulin dose by 91,67 , and $60 \%$ of patients, respectively [42]. A multinational study (China, France, Germany, Japan, Spain, Turkey, the UK, and the US) found that fear of weight gain was an additional factor reported by patients with T2D for insulin omission/treatment nonadherence [43]. When 530 insulintreated patients included in the study were asked to select their top three reasons for being nonadherent, the following were all reported: being too busy (18.9\%), travelling (16.2\%), skipped meal $(15.0 \%)$, stress or emotional problems $(11.7 \%)$, embarrassment of injecting in public $(9.7 \%)$, the challenge of injecting at the same time every day $(9.4 \%)$, forgot $(7.4 \%)$, too many injections (6.0\%), avoidance of weight gain $(4.0 \%)$, regimen was too complicated $(3.8 \%)$, and injections were too painful (2.6\%) [43].

Insulin-injection frequency has been shown to be inversely related to treatment persistence and adherence. A study in the US suggested a possible influence of insulin-injection frequency on treatment persistence [44]. Initiating insulin with once-daily insulin glargine rather than a twice-daily premixed insulin analogue was associated with increased treatment persistence $\quad(55.9 \%$ vs $45.4 \%$, respectively; $P<0.0001$ ), whereas treatment adherence was similar between groups (adjusted MPR 0.66 vs 0.64 , respectively; $P=0.19$ ) [44]. In a UK study, treatment adherence was significantly higher for patients with T2D requiring only 1 injection per day compared with patients requiring 4 injections per day $(78.3 \%$ vs $60.8 \%$, respectively; $P<0.0001$ ) [45]. Furthermore, in a review of mostly US studies (13 of 17), rigidity of treatment regimen was a factor identified as a patient-perceived barrier to treatment adherence [14].

A database analysis conducted across 6 European countries (Belgium, France, Germany, the Netherlands, Sweden, and the UK) examined real-world treatment patterns in patients with T2D initiating GLP-1 RAs [46]. At 1 year, among patients initiating exenatide, treatment modification (including discontinuation, up- or down-titration, and switching therapy) was higher for patients initiating a twice-daily regimen (84.1-93.8\% across countries) than for those initiating once-weekly exenatide (53.3-73.4\%). Across countries, although treatment patterns varied per country and according to the specific GLP-1 RA prescribed, treatment persistence (continuation of the index therapy) at 1 year was generally highest among patients initiating a once-weekly regimen, followed by a once-daily regimen, and then a twice-daily regimen. Other real-world data similarly show improved persistence and adherence among patients who were administered once-weekly exenatide compared to once-daily liraglutide $[47,48]$.

\section{IMPACT OF TREATMENT PERSISTENCE ON CLINICAL AND ECONOMIC OUTCOMES}

Lack of treatment persistence is associated with a lower likelihood of achieving the target HbA1c concentration and maintaining glycaemic control. In a large US study including $>56,000$ veterans with T2D, patients reported as having good treatment persistence with OADs over the 12 months following treatment initiation were more likely than nonpersisting patients to achieve the HbA1c goal of $\leq 7.0 \% \quad(\mathrm{RR} 1.07 ; 95 \%$ CI $1.06-1.09$ ) [49].

A pooled analysis of data from 3 US-based retrospective studies in patients with T2D who had previously been treated with OADs and who were initiated on basal insulin analogues (insulin glargine or insulin detemir) evaluated the consequences of treatment persistence/ nonpersistence over a 1-year follow-up period [50]. Patients who were persistent with 
treatment compared with nonpersistent patients had significantly lower average HbA1c levels at 1 year $(8.26 \%$ vs $8.46 \%$, respectively; $P=0.0260)$ and significantly greater average HbA1c reductions $(-1.15 \%$ vs $-0.86 \%$, respectively; $P=0.0078$ ), with no significant difference in hypoglycaemic events. Higher insulin treatment persistence was also associated with lower healthcare utilization [50]. Given these beneficial outcomes of treatment persistence, it does raise the question as to whether uncontrolled hyperglycaemia is part of a feedback loop involving not only persistence but also clinical inertia. Thus, intensifying treatment to improve glycaemic control might lead to improved persistence.

Treatment persistence in T2D is associated with decreased healthcare utilization and costs. In a US study, acute care (hospitalization and emergency department [ED]) costs were shown to be $9.6 \%$ higher among patients with T2D who discontinued insulin therapy (basal or mixture) early compared with patients who persisted with treatment beyond the first 90 days $(P<0.001)$ [51]. A German study reported that persisting on basal insulin glargine beyond the first 90 days, compared with switching insulins, was associated with significantly lower diabetes-related prescription costs (74EUR less) $(P=0.0780)$ [52].

Lack of treatment persistence with antidiabetes therapy is also linked to a higher likelihood of morbidity, mortality, and poorer health status [53].

\section{IMPACT OF TREATMENT ADHERENCE ON CLINICAL AND ECONOMIC OUTCOMES}

Medication adherence has also been shown to be associated with improved glycaemic control in patients with T2D. A retrospective cohort study in the US showed that patients with T2D who were considered adherent to a GLP-1 RA (PDC $\geq 0.80$ ) had a significantly greater reduction in HbA1c than nonadherent patients and were more likely to have a reduction in HbA1c of $\geq 1.0 \%$ [54]. Conversely, poor adherence to antidiabetes therapy is associated with failure to achieve HbA1c targets in the real-world setting. Using the four-item Morisky Medication Adherence Scale (a validated instrument for which higher scores indicate poorer adherence), patients who self-reported that they forgot to take medications at baseline were shown to have an absolute HbA1c increase of $0.43 \%$ after 6 months of treatment with insulin and/or OADs [55]. In another study, a one-point increase in the eight-item Morisky Medication Adherence Scale was found to be associated with a $0.21 \%$ increase in $\mathrm{HbA} 1 \mathrm{c}$ in patients with T2D using basal insulin analogues [56].

Poor medication adherence has been identified as a key reason why the effectiveness of antidiabetes therapy in the real-world setting is substantially less than the efficacy reported in randomized controlled clinical trials (RCTs) [57]. In an analysis of real-world data from patients with T2D initiating treatment with a GLP-1 RA $(n=221)$ or a DPP-4 inhibitor $(n=652)$, significantly smaller reductions in HbA1c were reported at 1 year in the real-world setting than had been reported in RCTs after the same period for both GLP-1 RAs $(-0.52 \%$ vs $-1.30 \%$, respectively; $P<0.01$ ) and DPP-4 inhibitors $(-0.51 \%$ vs $-0.68 \%$, respectively; $P=0.01$ ) [57]. The authors attributed approximately three-quarters of these gaps to patient treatment adherence rates, which were 29\% with GLP-1 RAs and 37\% with DPP-4 inhibitors in the real-world analysis, compared with 95\% for both drug classes in the RCTs. The study underscored the urgent need to effectively improve treatment adherence among patients with T2D in the real world. Additionally, it alludes to the impact of the route of administration on treatment adherence.

Hyperglycaemia and long-term complications of T2D are associated with poor adherence to antidiabetes medications and have important clinical consequences for the patient [58]. A narrative review of studies published in English and Spanish identified several studies linking poor adherence to T2D therapies with increased risks of morbidity and premature mortality [58].

A large UK study of almost 16,000 patients with T2D who were treated with a combination of OADs and insulin found that after adjustment for confounding factors, medication 
nonadherence was independently associated with increased all-cause mortality (hazard ratio 1.579; 95\% CI 1.167-2.135) [35].

A systematic review by Banerji et al. [59] investigating the impact of glycaemic control and medication treatment adherence on clinical and economic outcomes in patients with T2D confirmed the wealth of data linking poor medication treatment adherence to increased medical resource utilization and healthcare costs.

A 2005 analysis of the impact of medication treatment adherence in diabetes on healthcare costs, within a large population-based sample of patients aged $<65$ years, estimated that a $20 \%$ increase in medication adherence, while increasing the average drug-utilization costs by 177 USD per patient per year, would result in a disease-related medical-cost reduction of 1251 USD per patient per year [60]. This would result in a net saving of 1074 USD per patient per year [60].

Real-world studies in the US have shown that total healthcare expenditures for patients with T2D who utilized insulin pens decreased with improvements in adherence. Significant decreases in ED visits (odds ratio [OR] 0.44; 95\% CI $0.21-0.92 ; P<0.05)$ and physician visits (OR $0.39 ; 95 \%$ CI $0.24-0.64 ; P<0.05)$ have been reported after conversion from vial and syringe to pen administration of insulin therapy. These are associated with total mean all-cause treatment costs reductions of 1590 USD per patient per year [61]. Additionally, a large study of 23,362 patients with T2D who used an insulin pen found that the average per patient per year healthcare expenditure was $9.4 \%$ lower for patients in the most adherent (MPR 0.81-1.00) compared with the least adherent (MPR $0.00-0.20)$ groups $(23,839$ USD vs 26,310 USD, respectively; $P=0.007$ ) [62].

Other US analyses investigating the economic consequences of treatment nonadherence have shown increased resource utilization and healthcare costs associated with poor adherence. DiBonaventura et al. [56] found that, for patients with T2D using basal insulin analogues, each one-point increase in treatment nonadherence on the eight-item Morisky Medication Adherence Scale was associated with a
$4.6,20.4$, and $20.9 \%$ increase in the number of physician visits, ED visits, and hospitalizations, respectively. Encinosa et al. [63] reported that, in non-elderly patients with T2D, an increase in treatment adherence to OADs from 50\% to $100 \%$ resulted in a $23.3 \%$ reduction in the rate of hospitalization and a $46.2 \%$ reduction in ED visits, leading to cost savings of 866 USD per patient and a cost offset of 1.14 USD for every 1.00 USD spent on diabetic drugs.

Other studies have explored the potential impact of treatment adherence on diabetes complications. A retrospective database analysis of new OAD users found that good adherence (defined as MPR $\geq 0.8$ ) was associated with significantly reduced risk of a new microvascular or macrovascular diabetes complication (adjusted hazard ratio 0.96 ; 95\% CI 0.92-1.00; $P=0.05$ ) [64]. Initial adherence appears to be important, with another retrospective cohort study observing that during the first 5 years of OAD treatment, those who were initially nonadherent to therapy were more likely to experience myocardial infarction, ischaemic stroke, or death [65].

This review is limited by the inclusion of studies that the authors regard as being most pertinent to the central review objectives, identified within a relatively short timeframe. It is not a comprehensive review of the field, nor is it a systematic review. One consequent limitation is that no studies have been included concerning the use of long-acting insulin degludec. However, we know of no data suggesting any difference between insulin glargine 300 units/mL and insulin degludec regarding the quality of adherence to insulin therapy or the rate of persistence. Because reimbursement issues are very complex and differ widely according to the country and healthcare system, it has not been discussed here.

\section{CONCLUSION}

For patients with T2D, poor persistence with and adherence to antidiabetes medications can increase the risk of long-term complications, leading to poorer health status and an increase in healthcare resource utilization and costs. A 
clear unmet need remains in T2D for therapies that improve treatment persistence and adherence compared with currently available treatments, thereby positively impacting clinical and economic outcomes.

Several approaches to improving treatment persistence and adherence have been suggested, including: reducing treatment complexity (e.g. using fixed-dose combination therapy that decreases the frequency of administration [24, 35], implantable therapies for drug delivery); developing medications with an improved safety profile (e.g. lower risk of weight gain, hypoglycaemia, gastrointestinal side effects $[20,35])$; enhancing educational initiatives; and improving communication (e.g. telemedicine approaches, including websites and electronic records) [58]. In addition, the impact of treatment persistence and adherence on disease management must be stressed at the time of treatment initiation [65].

Lack of treatment persistence and treatment nonadherence are highly prevalent among patients with T2D, and there is a global need to address the complexity of antidiabetes therapy and simplify treatment regimens. Fixed-ratio combinations offer a prospective solution to overcome barriers to injectable therapy and treatment intensification, and they would thereby increase treatment persistence and adherence due to less complex regimens requiring fewer daily injections.

\section{ACKNOWLEDGEMENTS}

Funding. This review and the journal's article processing charges were funded by Sanofi. All authors had full access to the information used to compile this work and take complete responsibility for the integrity and accuracy of this review.

Medical Writing and Editorial Assistance. The authors received medical writing/ editorial support in the preparation of this manuscript from Grace Richmond, PhD, of Excerpta Medica, funded by Sanofi.
Authorship. All named authors meet the International Committee of Medical Journal Editors (ICMJE) criteria for authorship for this article, take responsibility for the integrity of the work as a whole, and have given their approval for this version to be published.

Authorship Contributions. Bruno Guerci was involved in data analysis or interpretation, critical revision, and final approval of the manuscript, and is accountable for accuracy and integrity. Neha Chanan and Simarjeet Kaur were involved in data acquisition, data analysis or interpretation, critical revision, and final approval of the manuscript, and are accountable for accuracy and integrity. Juan Guillermo Jasso-Mosqueda and Elisheva Lew were involved in concept or design, data analysis or interpretation, critical revision, and final approval of the manuscript, and are accountable for accuracy and integrity.

Disclosures. Bruno Guerci has served on advisory panels/boards for Sanofi, Eli Lilly, Novo Nordisk, Novartis, GSK, MSD, Boehringer Ingelheim, AstraZeneca, Abbott, Medtronic, and Roche Diagnostics; has served as a clinical investigator for Sanofi, Eli Lilly, Novo Nordisk, GSK, BMS, AstraZeneca, Medtronic, Abbott, Roche Diagnostics, MSD, Novartis, Janssen, and Boehringer Ingelheim; and has received research support from Medtronic, VitalAire, Sanofi, Eli Lilly, and Novo Nordisk. Neha Chanan is an employee of Parexel Access Consulting, which received funding from Sanofi to perform this analysis. Simarjeet Kaur is an employee of Parexel Access Consulting, which received funding from Sanofi to perform this analysis. Juan Guillermo Jasso-Mosqueda is an employee of Sanofi. Elisheva Lew is an employee of Sanofi.

Compliance with Ethics Guidelines. This review is based on previously conducted studies and does not contain any studies with human participants or animals performed by any of the authors.

Open Access. This article is distributed under the terms of the Creative Commons 
Attribution-NonCommercial 4.0 International License (http://creativecommons.org/licenses/ by-nc/4.0/), which permits any noncommercial use, distribution, and reproduction in any medium, provided you give appropriate credit to the original author(s) and the source, provide a link to the Creative Commons license, and indicate if changes were made.

\section{REFERENCES}

1. Cramer JA, Roy A, Burrell A, et al. Medication compliance and persistence: terminology and definitions. Value Health. 2008;11(1):44-7.

2. Martin BC, Wiley-Exley EK, Richards S, Domino ME, Carey TS, Sleath BL. Contrasting measures of adherence with simple drug use, medication switching, and therapeutic duplication. Ann Pharmacother. 2009;43(1):36-44.

3. American Diabetes Association. 6. Glycemic targets: in standards of medical care in diabetes-2017. Diabetes Care. 2017;40(Suppl 1):S48-56.

4. Inzucchi SE, Bergenstal RM, Buse JB, et al. Management of hyperglycaemia in type 2 diabetes, 2015: a patient-centred approach. Update to a position statement of the American Diabetes Association and the European Association for the Study of Diabetes. Diabetologia. 2015;58(3):429-42.

5. Nau DP. Recommendations for improving adherence to type 2 diabetes mellitus therapy: focus on optimizing oral and non-insulin therapies. Am J Manag Care. 2012;18(3 Suppl):S49-54.

6. Jermendy G, Kiss Z, Rokszin G, Abonyi-Toth Z, Wittmann I, Kempler P. Persistence to treatment with novel antidiabetic drugs (dipeptidyl peptidase4 inhibitors, sodium-glucose co-transporter- 2 inhibitors, and glucagon-like peptide-1 receptor agonists) in people with type 2 diabetes: a nationwide cohort study. Diabetes Ther. 2018;9(5):2133-41.

7. Miao R, Wei W, Baser O, Xie L. Real world outcomes of adding rapid-acting insulin versus switching to analog premix insulin among US patients with type 2 diabetes treated with insulin glargine. Patient Prefer Adherence. 2013;7:951-60.

8. Yavuz DG, Ozcan S, Deyneli O. Adherence to insulin treatment in insulin-naïve type 2 diabetic patients initiated on different insulin regimens. Patient Prefer Adherence. 2015;9:1225-31.
9. Pan F, Chernew ME, Fendrick AM. Impact of fixeddose combination drugs on adherence to prescription medications. J Gen Intern Med. 2008;23(5):611-4.

10. Mashitani T, Hayashino Y, Okamura S, et al. Diabetes treatment-related quality of life is associated with levels of self-care activities in insulin injection among Japanese patients with type 2 diabetes: Diabetes Distress and Care Registry at Tenri (DDCRT 8). Acta Diabetol. 2015;52(4):639-47.

11. Mashitani T, Hayashino $\mathrm{Y}$, Okamura $\mathrm{S}$, et al. Patient-reported adherence to insulin regimen is associated with glycemic control among Japanese patients with type 2 diabetes: Diabetes Distress and Care Registry at Tenri (DDCRT 8). Diabetes Res Clin Pract. 2013;100(2):189-94.

12. Brod M, Rana A, Barnett AH. Adherence patterns in patients with type 2 diabetes on basal insulin analogues: missed, mistimed and reduced doses. Curr Med Res Opin. 2012;28(12):1933-46.

13. Farmer A, Kinmonth AL, Sutton S. Measuring beliefs about taking hypoglycaemic medication among people with type 2 diabetes. Diabet Med. 2006;23(3):265-70.

14. Davies MJ, Gagliardino JJ, Gray LJ, Khunti K, Mohan V, Hughes R. Real-world factors affecting adherence to insulin therapy in patients with type 1 or type 2 diabetes mellitus: a systematic review. Diabet Med. 2013;30(5):512-24.

15. Carls GS, Tan R, Zhu JY, et al. Real-world weight change among patients treated with glucagon-like peptide- 1 receptor agonist, dipeptidyl peptidase- 4 inhibitor and sulfonylureas for type 2 diabetes and the influence of medication adherence. Obes Sci Pract. 2017;3(3):342-51.

16. Ali MM, Aung KT, Young M, et al. Different insulin initiation regimens in patients with type 2 diabetes-a review article. Int J Diabetes Clin Res. 2018;5:083.

17. Downie M, Kilov G, Wong J. Initiation and intensification strategies in type 2 diabetes management: a comparison of basal plus and premix regimens. Diabetes Ther. 2016;7(4):641-57.

18. Levin PA, Wei W, Zhou S, Xie L, Baser O. Outcomes and treatment patterns of adding a third agent to 2 OADs in patients with type 2 diabetes. J Manag Care Spec Pharm. 2014;20(5):501-12.

19. Bron M, Marynchenko M, Yang H, Yu AP, Wu EQ. Hypoglycemia, treatment discontinuation, and costs in patients with type 2 diabetes mellitus on oral antidiabetic drugs. Postgrad Med. 2012;124(1):124-32. 
20. Gelhorn HL, Stringer SM, Brooks A, et al. Preferences for medication attributes among patients with type 2 diabetes mellitus in the UK. Diabetes Obes Metab. 2013;15(9):802-9.

21. Lopez JM, Annunziata K, Bailey RA, Rupnow MF, Morisky DE. Impact of hypoglycemia on patients with type 2 diabetes mellitus and their quality of life, work productivity, and medication adherence. Patient Prefer Adherence. 2014;8:683-92.

22. Walz L, Pettersson B, Rosenqvist U, Deleskog A, Journath $G$, Wändell P. Impact of symptomatic hypoglycemia on medication adherence, patient satisfaction with treatment, and glycemic control in patients with type 2 diabetes. Patient Prefer Adherence. 2014;8:593-601.

23. Lokhandwala T, Smith N, Sternhufvud C, Sörstadius E, Lee WC, Mukherjee J. A retrospective study of persistence, adherence, and health economic outcomes of fixed-dose combination vs. loose-dose combination of oral anti-diabetes drugs. J Med Econ. 2016;19(3):203-12.

24. Bajaj HS, Ye C, Jain E, Venn K, Stein E, Aronson R. Glycemic improvement with a fixed-dose combination of DPP-4 inhibitor + metformin in patients with type 2 diabetes (GIFT study). Diabetes Obes Metab. 2018;20(1):195-9.

25. Venditti EM, Tan K, Chang N, et al. Barriers and strategies for oral medication adherence among children and adolescents with type 2 diabetes. Diabetes Res Clin Pract. 2018;139:24-31.

26. Shiyanbola OO, Unni E, Huang YM, Lanier C. The association of health literacy with illness perceptions, medication beliefs, and medication adherence among individuals with type 2 diabetes. Res Soc Adm Pharm. 2018;14(9):824-30.

27. He X, Chen L, Wang $\mathrm{K}, \mathrm{Wu} \mathrm{H}, \mathrm{Wu}$ J. Insulin adherence and persistence among Chinese patients with type 2 diabetes: a retrospective database analysis. Patient Prefer Adherence. 2017;11:237-45.

28. Roussel R, Charbonnel B, Behar M, Gourmelen J, Emery C, Detournay B. Persistence with insulin therapy in patients with type 2 diabetes in France: an insurance claims study. Diabetes Ther. 2016;7(3):537-49.

29. Reynolds SL, Zhou S, Uribe C, Li Y. Impact of insulin delivery systems in elderly patients with type 2 diabetes. Am J Pharm Benefits. $2015 ; 7: 222-31$.

30. Miao R, Wei W, Lin J, Xie L, Baser O. Does device make any difference? A real-world retrospective study of insulin treatment among elderly patients with type 2 diabetes. J Diabetes Sci Technol. 2014;8(1):150-8.

31. Xie L, Wei W, Pan C, Du J, Baser O. A real-world study of patients with type 2 diabetes initiating basal insulins via disposable pens. Adv Ther. 2011;28(11):1000-11.

32. Wei W, Zhou S, Miao R, et al. Much ado about nothing? A real-world study of patients with type 2 diabetes switching basal insulin analogs. Adv Ther. 2014;31(5):539-60.

33. Pscherer S, Chou E, Dippel FW, Rathmann W, Kostev K. Treatment persistence after initiating basal insulin in type 2 diabetes patients: a primary care database analysis. Prim Care Diabetes. 2015;9(5):377-84.

34. Chou E, Dippel FW, Chung H. Treatment persistence and switch in German patients with type 2 diabetes mellitus (T2DM) after initiating basal insulin. Paper presented at: American Diabetes Association 74th Scientific Sessions 2014; 2014 June 13-17; San Francisco, CA, USA.

35. Garcia-Pérez LE, Alvarez M, Dilla T, Gil-Guillén V, Orozco-Beltrán D. Adherence to therapies in patients with type 2 diabetes. Diabetes Ther. 2013;4(2):175-94.

36. Meece J. Basal insulin intensification in patients with type 2 diabetes: a review. Diabetes Ther. 2018;9(3):877-90.

37. Jarab AS, Almrayat R, Alqudah S, et al. Predictors of non-adherence to pharmacotherapy in patients with type 2 diabetes. Int $\mathrm{J}$ Clin Pharm. 2014;36(4):725-33.

38. Blüher M, Kurz I, Dannenmaier S, Dworak M. Pill burden in patients with type 2 diabetes in Germany: subanalysis from the prospective, noninterventional PROVIL study. Clin Diabetes. 2015;33(2):55-61.

39. Sussman M, Griffiths R, Wang H, Tong L, KitioDschassi B. Compliance with treatment following initiation of free combination therapy with basal insulin and glp-1 among patients with diabetes. Paper presented at: American Diabetes Association 76th Scientific Sessions 2016; 2016 June 10-14; New Orleans, LA, USA.

40. Nicolucci A, Kovacs Burns K, Holt RI, et al. Diabetes Attitudes, Wishes and Needs Second Study (DAWN2 ${ }^{\mathrm{TM}}$ ): cross-national benchmarking of diabetes-related psychosocial outcomes for people with diabetes. Diabet Med. 2013;30(7):767-77.

41. Reach G, Consoli SM, Halimi S, et al. The multinational second diabetes, attitudes, wishes and 
needs study: results of the French survey. Patient Prefer Adherence. 2015;9:289-97.

42. Ampudia-Blasco FJ, Galan M, Brod M. A cross-sectional survey among patients and prescribers on insulin dosing irregularities and impact of mild (self-treated) hypoglycemia episodes in Spanish patients with type 2 diabetes as compared to other European patients. Endocrinol Nutr. 2014;61(8):426-33.

43. Peyrot M, Barnett AH, Meneghini LF, SchummDraeger PM. Insulin adherence behaviours and barriers in the multinational Global Attitudes of Patients and Physicians in Insulin Therapy study. Diabet Med. 2012;29(5):682-9.

44. Baser O, Tangirala K, Wei W, Xie L. Real-world outcomes of initiating insulin glargine-based treatment versus premixed analog insulins among US patients with type 2 diabetes failing oral antidiabetic drugs. Clinicoecon Outcomes Res. 2013;5:497-505.

45. Donnelly LA, Morris AD, Evans JM. Adherence to insulin and its association with glycaemic control in patients with type 2 diabetes. QJM. 2007;100(6):345-50.

46. Divino V, DeKoven M, Khan FA, Boye KS, Sapin H, Norrbacka K. GLP-1 RA treatment patterns among type 2 diabetes patients in five European countries. Diabetes Ther. 2017;8(1):115-28.

47. Qiao Q, Ouwens MJ, Grandy S, Johnsson K, Kostev K. Adherence to GLP-1 receptor agonist therapy administered by once-daily or once-weekly injection in patients with type 2 diabetes in Germany. Diabetes Metab Syndr Obes. 2016;9:201-5.

48. Yu M, Xie J, Fernandez Lando L, Kabul S, Swindle RW. Liraglutide versus exenatide once weekly: persistence, adherence, and early discontinuation. Clin Ther. 2016;38(1):149-60.

49. Kim N, Agostini JV, Justice AC. Refill adherence to oral hypoglycemic agents and glycemic control in veterans. Ann Pharmacother. 2010;44(5):800-8.

50. Wei W, Pan C, Xie L, Baser O. Real-world insulin treatment persistence among patients with type 2 diabetes. Endocr Pract. 2014;20(1):52-61.

51. Ascher-Svanum H, Lage MJ, Perez-Nieves M, et al. Early discontinuation and restart of insulin in the treatment of type 2 diabetes mellitus. Diabetes Ther. 2014;5(1):225-42.

52. Anderten H, Dippel FW, Kostev K. Early discontinuation and related treatment costs after initiation of basal insulin in type 2 diabetes patients: a
German primary care database analysis. J Diabetes Sci Technol. 2015;9(3):644-50.

53. Khunti K, Seidu S, Kunutsor S, Davies M. Association between adherence to pharmacotherapy and outcomes in type 2 diabetes: a meta-analysis. Diabetes Care. 2017;40(11):1588-96.

54. Buysman EK, Liu F, Hammer M, Langer J. Impact of medication adherence and persistence on clinical and economic outcomes in patients with type 2 diabetes treated with liraglutide: a retrospective cohort study. Adv Ther. 2015;32(4):341-55.

55. Aikens JE, Piette JD. Longitudinal association between medication adherence and glycaemic control in type 2 diabetes. Diabet Med. 2013;30(3):338-44.

56. DiBonaventura M, Wintfeld N, Huang J, Goren A. The association between nonadherence and glycated hemoglobin among type 2 diabetes patients using basal insulin analogs. Patient Prefer Adherence. 2014;8:873-82.

57. Carls GS, Tuttle E, Tan RD, et al. Understanding the gap between efficacy in randomized controlled trials and effectiveness in real-world use of GLP-1 RA and DPP- 4 therapies in patients with type 2 diabetes. Diabetes Care. 2017;40(11):1469-78.

58. Currie CJ, Peyrot M, Morgan CL, et al. The impact of treatment non-compliance on mortality in people with type 2 diabetes. Diabetes Care. 2012;35(6):1279-84.

59. Banerji MA, Dunn JD. Impact of glycemic control on healthcare resource utilization and costs of type 2 diabetes: current and future pharmacologic approaches to improving outcomes. Am Health Drug Benefits. 2013;6(7):382-92.

60. Sokol MC, McGuigan KA, Verbrugge RR, Epstein RS. Impact of medication adherence on hospitalization risk and healthcare cost. Med Care. 2005;43(6):521-30.

61. Lee WC, Balu S, Cobden D, Joshi AV, Pashos CL. Medication adherence and the associated healtheconomic impact among patients with type 2 diabetes mellitus converting to insulin pen therapy: an analysis of third-party managed care claims data. Clin Ther. 2006;28(10):1712-25.

62. Chandran A, Bonafede MK, Nigam S, Saltiel-Berzin R, Hirsch LJ, Lahue BJ. Adherence to insulin pen therapy is associated with reduction in healthcare costs among patients with type 2 diabetes mellitus. Am Health Drug Benefits. 2015;8(3):148-58.

63. Encinosa WE, Bernard D, Dor A. Does prescription drug adherence reduce hospitalizations and costs? 
The case of diabetes. Adv Health Econ Health Serv Res. 2010;22:151-73.

64. Simpson SH, Lin M, Eurich DT. Medication adherence affects risk of new diabetes complications: a cohort study. Ann Pharmacother. 2016;50(9): 741-6.
65. Gatwood JD, Chisholm-Burns M, Davis R, et al. Differences in health outcomes associated with initial adherence to oral antidiabetes medications among veterans with uncomplicated type 2 diabetes: a 5-year survival analysis. Diabet Med. 2018;35(11):1571-9. 